\title{
Efficacy of cetuximab-based chemotherapy in metastatic colorectal cancer according to RAS and BRAF mutation subgroups: A meta-analysis
}

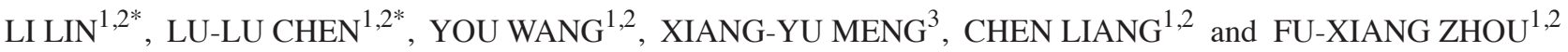 \\ ${ }^{1}$ Hubei Cancer Clinical Study Center, Hubei Key Laboratory of Tumor Biological Behaviors; \\ ${ }^{2}$ Department of Radiation Oncology and Medical Oncology; ${ }^{3}$ Center for Evidence-Based and Translational Medicine, \\ Zhongnan Hospital of Wuhan University, Wuhan, Hubei 430071, P.R. China
}

Received September 17, 2015; Accepted February 24, 2016

DOI: $10.3892 / \operatorname{mco} .2016 .836$

\begin{abstract}
The epidermal growth factor receptor (EGFR)-targeting monoclonal antibody, cetuximab, has been added to standard chemotherapy regimens for treating metastatic colorectal cancer (mCRC). However, the efficacy of adding cetuximab to chemotherapy regimens for patients of differing genetic backgrounds remains controversial. The present study aimed to investigate the efficacy of adding cetuximab to chemotherapeutic regimens in subgroups of patients defined according to the RAS and BRAF mutation status in the first-line treatment of patients with mCRC. A systematic literature search was performed in databases (including PubMed, Embase, the Cochrane library, the American Society of Clinical Oncology and the European Society For Medical Oncology) up to August 2015. Randomized controlled trials analyzing overall survival (OS) and progression-free survival (PFS) in mCRC treated with cetuximab, and grouped by RAS and BRAF mutation status, were identified. The major outcome measures were hazard ratios (HRs). Pooled HRs were calculated using fixed- or random-effects models, according to the magnitude of the heterogeneity. A total of nine studies met the inclusion criteria. Use of cetuximab was significantly associated with longer OS in KRAS exon 2 wild-type tumors [HR=0.87, 95\% confidence interval $(\mathrm{CI})=0.79-0.96, \mathrm{Z}=2.91, \mathrm{P}=0.004]$ and wild-type KRAS/RAS (in exons 2, 3 and 4 of KRAS and exons 2, 3 and 4 of an associated gene, NRAS; HR=0.72,
\end{abstract}

Correspondence to: Professor Fu-Xiang Zhou, Department of Radiation Oncology and Medical Oncology, Zhongnan Hospital of Wuhan University, 169 Donghu Road, Wuhan, Hubei 430071, P.R. China

E-mail: happyzhoufx@sina.com

${ }^{*}$ Contributed equally

Key words: colorectal neoplasms, RAS, BRAF, cetuximab, meta-analysis
$95 \% \mathrm{CI}=0.60-0.85, \mathrm{Z}=3.74, \mathrm{P}=0.0002)$. No significant differences in OS and PFS were identified between KRAS exon 2 mutations and tumors with the other RAS mutations (in exons 3 and 4 of KRAS and exons 2, 3 and 4 of an associated gene, NRAS). The meta-analysis demonstrated that cetuximab-based chemotherapeutic regimens led to a marked improvement in OS in patients with $\mathrm{mCRC}$ who lacked any RAS mutations (either KRAS exon 2 or any other RAS mutation). By contrast, the subgroup analyses revealed no evident PFS or OS benefit in using cetuximab for patients with any RAS mutation. Taken together, the evidence indicates that cetuximab should only be used for mCRC patients with the wild-type RAS gene. Some benefits were observed in patients with wild-type KRAS/BRAF who received cetuximab-based chemotherapy, even though there were insufficient data to perform meta-analysis with the BRAF mutation status.

\section{Introduction}

Colorectal cancer $(\mathrm{CRC})$ remains one of the most common causes of cancer-associated mortality (1). Over the past decades, novel therapeutic options have been introduced as treatments for metastatic CRC (mCRC). A combination of chemotherapy with targeted therapy has been regarded as a standard first-line treatment plan (2). Cetuximab, a monoclonal antibody, targets the extracellular domain of the epidermal growth factor receptor (EGFR), exerting an important role in the treatment of patients with mCRC. However, studies concerning the effects of cetuximab-based chemotherapy as a first-line mCRC treatment have demonstrated divergent results (2-4). RAS family proteins (including KRAS and NRAS) exert important roles in EGFR-mediated intracellular signaling cascades. Mutations in RAS genes (occurring at loci in exons 2, 3 and 4) are often identified in mCRC, and the most common of these is KRAS exon 2 (codon 12/13). In several previous studies, including CRYSTAL phase III (2) and OPUS phase II (3) randomized clinical trials (RCTs), the use of cetuximab was limited to treatment for $\mathrm{mCRC}$ patients with the wild-type KRAS gene. However, as revealed in the COIN (4) and NORDIC-VII (5) trials, cetuximab is not always effective in KRAS wild-type patients. The previous studies 
evaluating anti-EGFR monoclonal antibodies in mCRC were amended to focus on KRAS exon 2-selected populations, either retrospectively evaluating outcomes in KRAS exon 2 wild-type patients or prospectively enrolling KRAS exon 2 wild-type patients (6-8). Mutations of KRAS predominantly lie in codons 12 and 13 of exon 2 . However, additionally, there are $\sim 5 \%$ of CRC patients who have mutations in KRAS exons 3 or 4 , usually at codons 61 or 146 , and a further $\sim 5 \%$ of patients with CRC with mutations in NRAS exons 2, 3 or 4. Furthermore, almost $10 \%$ of patients with CRC have mutations in BRAF (9). It was suggested that other mutations, including ones in RAS (in exons 3 and 4 of KRAS and exons 2, 3 and 4 of NRAS) and BRAF (KRAS exon 2), in patients with wild-type mCRC patients may exert similar negative effects on the efficacy of EGFR-targeted therapy $(2,3,10)$. Previously, data from certain retrospective analyses of several phase III trials indicated that all RAS mutations were regarded as a negative predictive factor of anti-EGFR therapy (11). Similar analyses performed for patients with BRAF mutations, mutually exclusive of RAS mutations, demonstrated a consistently poor prognosis, regardless of the treatment strategy employed $(11,12)$.

Therefore, the meta-analysis in the present study was performed to assess the efficacy of adding cetuximab to chemotherapies in the first-line treatment of $\mathrm{mCRC}$, and to investigate the prognosis and outcomes for cetuximab-based chemotherapy in populations of differing RAS and BRAF mutation status.

\section{Materials and methods}

Search strategy. Two independent investigators (L. Lin and L.L. Chen) searched electronic databases, including PubMed, Embase, the Cochrane library, the American Society of Clinical Oncology and the European Society For Medical Oncology, up to August 2015. The following search items were used: 'Colorectal Neoplasms' AND 'cetuximab' AND 'Clinical Trial' AND 'Ras', and relevant Medical Subject Heading ('MeSH') terms were utilized. References cited in the publications were searched to identify additional relevant studies. Additional articles that were missed from the search strategy were also searched after.

Eligibility criteria. Eligible studies had to meet the following criteria: i) the study was an RCT; ii) the patients had pathologically confirmed mCRC; iii) cetuximab-based chemotherapy was compared with chemotherapy \pm other targeted agents (e.g. bevacizumab) as the first-line treatment; iv) the outcomes of interest were survival according to the RAS and BRAF gene mutation status; v) the study either provided, or allowed for, the calculation of hazard ratios (HRs) with corresponding 95\% confidence intervals (CIs); and vi) only studies with full text were included. If duplicated or overlapped data were identified in multiple reports, the one with most comprehensive information was included. Studies with a single-arm design, or RCTs with arms all containing cetuximab, were excluded. Finally, studies not published in English were excluded.

Quality assessment. Two investigators (L. Lin and L.L. Chen) independently rated the quality of the retrieved studies. The risk of bias items recommended by The Cochrane Handbook for Systematic Reviews of Interventions (13) was selected.

Data extraction. Two investigators (L. Lin and L.L. Chen) independently extracted the following information from each study. Disagreements were revolved by consensus. From each of the eligible studies, the following information was collected: The first author's family name, publication year, treatment regimens, sample size, blind trial type, type of controls, HRs with corresponding $95 \%$ CIs or relevant data for HR, and the 95\% CI calculation for OS and/or PFS. The data were extracted separately according to the RAS and BRAF mutation status.

Statistical analysis. The efficacy of adding cetuximab to the chemotherapy regimen in the treatment of $\mathrm{mCRC}$, based on the data from RCTs, was assessed. The endpoints of interest in the pooled analysis were OS and PFS, and they were thus expressed by HRs with $95 \%$ CIs for each study. The association between the efficacy of adding cetuximab to the chemotherapy in the treatment of mCRC, and OS or PFS, was considered as a weighted average of the individual estimate of the HR in every included study, using the inverse variance method. The natural logarithms of the HRs (lnHRs) were considered to obey a normal distribution. If the HRs and the corresponding 95\% CIs were reported, lnHRs and the corresponding natural logarithms of the upper and lower limbs of the distribution were used as data points in the pooling analysis. A sensitivity analysis was also performed to examine the impact on the overall results (PFS and OS), depending on the heterogeneity between the included studies. Prior to the synthesis of the original data, $\mathrm{I}^{2}$ statistics were used to assess the homogeneity (13). Studies with an $\mathrm{I}^{2}$ of $25-50,50-75$ or $>75 \%$ were considered to have low, moderate or high heterogeneity, respectively (14). The pooled HRs were first calculated using the fixed-effects model. If there was high heterogeneity among the studies, the randomized-effects model was used. Subgroup analysis was performed according to the KRAS and BRAF gene type, with the aim of exploring important clinical differences among trials that could be expected to affect the magnitude of the treatment effect. $\mathrm{P}<0.05$ was considered to indicate a statistically significant difference. All the statistical tests in this meta-analysis were performed with Review Manager version 5.3 software (Revman; The Cochrane collaboration Oxford, United Kingdom). The findings of the present meta-analysis are shown in forest plots.

\section{Results}

Overview of the literature search and study characteristics. An overall total of 336 studies were retrieved initially after searching the databases and hand-searched articles. Of these articles, 14 full-text articles were evaluated in more detail, and a total of nine articles were included in the meta-analysis (Fig. 1). All studies included in the present study were considered to be of moderate quality, at least. Table I shows the primary characteristics of the nine studies, and the risk of bias items is shown in Fig. 2. In the present review, cetuximab-based chemotherapy was administered as a first-line treatment in patients with mCRC. Seven of the studies were open-label, randomized, multicenter phase III trials, and two of them 
Table I. Baseline characteristics of patients in the trials included in the meta-analysis.

\begin{tabular}{|c|c|c|c|c|c|}
\hline Authors, year & Clinical trial & $\begin{array}{c}\text { Type of } \\
\text { study }\end{array}$ & $\begin{array}{l}\text { Treatment } \\
\text { regimen }\end{array}$ & $\begin{array}{l}\text { Subgroup } \\
\text { analysis }\end{array}$ & Refs. \\
\hline Maughan et al, 2011 & COIN & Phase III & Oxa+FU vs. Oxa+FU+cet & KRAS BRAF & (4) \\
\hline Bokemeyer et al, 2011 & OPUS & Phase II & FOLFOX-4+cet vs. FOLFOX-4 & ITT KRAS BRAF & (3) \\
\hline Van Cutsem et al, 2011 & CRYSTAL & Phase III & FOLFIRI vs. FOLFIRI+cet & ITT KRAS BRAF & $(2)$ \\
\hline Tveit $^{\mathrm{a}}$ et al, 2012 & NORDIC-VII & Phase III & $\begin{array}{l}\text { A; Nordic FLOX } \\
\text { B: FLOX+cet } \\
\text { C: intermittent FLOX +cet }\end{array}$ & ITT KRAS & $(5)$ \\
\hline Stintzing et al, 2012 & AIO-0306 & Phase III & FOLFIRI+cet vs. FOLFIRI + bev & $\mathrm{ITT}^{\mathrm{b}}$ & $(17)$ \\
\hline Ye et al, 2013 & & Phase III & CT+cet vs. CT & $\operatorname{ITT}^{\mathrm{c}}$ & $(19)$ \\
\hline Heinemann et al, 2014 & FIRE-3 & Phase III & FOLFIRI +cet vs. FOLFORI +bev & ITT $^{c}$ KRAS RAS & $(18)$ \\
\hline Van Cutsem et al, 2015 & CRYSTAL & Phase III & FOLFIRI vs. FOLFIRI+cet & KRAS RAS & $(16)$ \\
\hline Bokemeyer et al, 2015 & OPUS & Phase II & FOLFOX-4+cet vs. FOLFOX-4 & KRAS RAS & $(20)$ \\
\hline
\end{tabular}

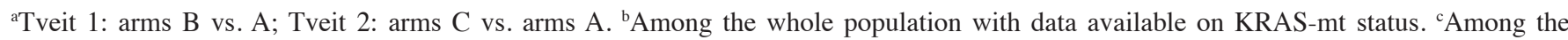
whole population with data available about KRAS-wt status. cet, cetuximab; bev; bevacizumab; Oxa + FU, oxaliplatin + fluoropyrimidine; FOLFOX-4, 5-fluoropyrimidine + folinic acid + oxaliplatin; FOLFIRI, fluorouracil + leucovorin + irinotecan; Nordic FLOX, fluorouracil/folinic acid and oxaliplatin; FLOX, oxaliplatin + fluoropyrimidine; CT, FOLFIRI (fluorouracil + leucovorin + irinotecan) or mFOLFOX6 (modified fluorouracil + leucovorin + oxaliplatin); ITT, intention-to-treat.

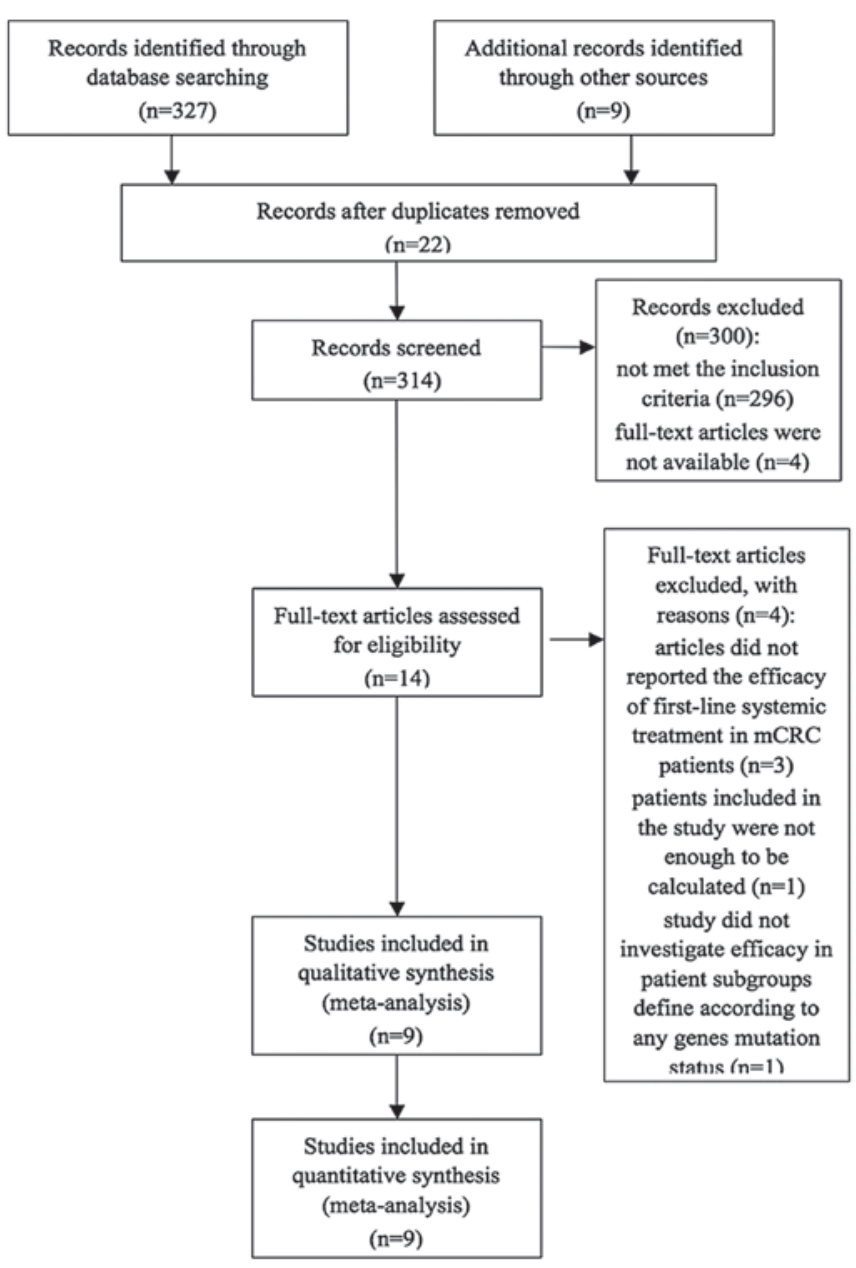

Figure 1. PRISMA flow chart of selection process to identify studies eligible for pooling.

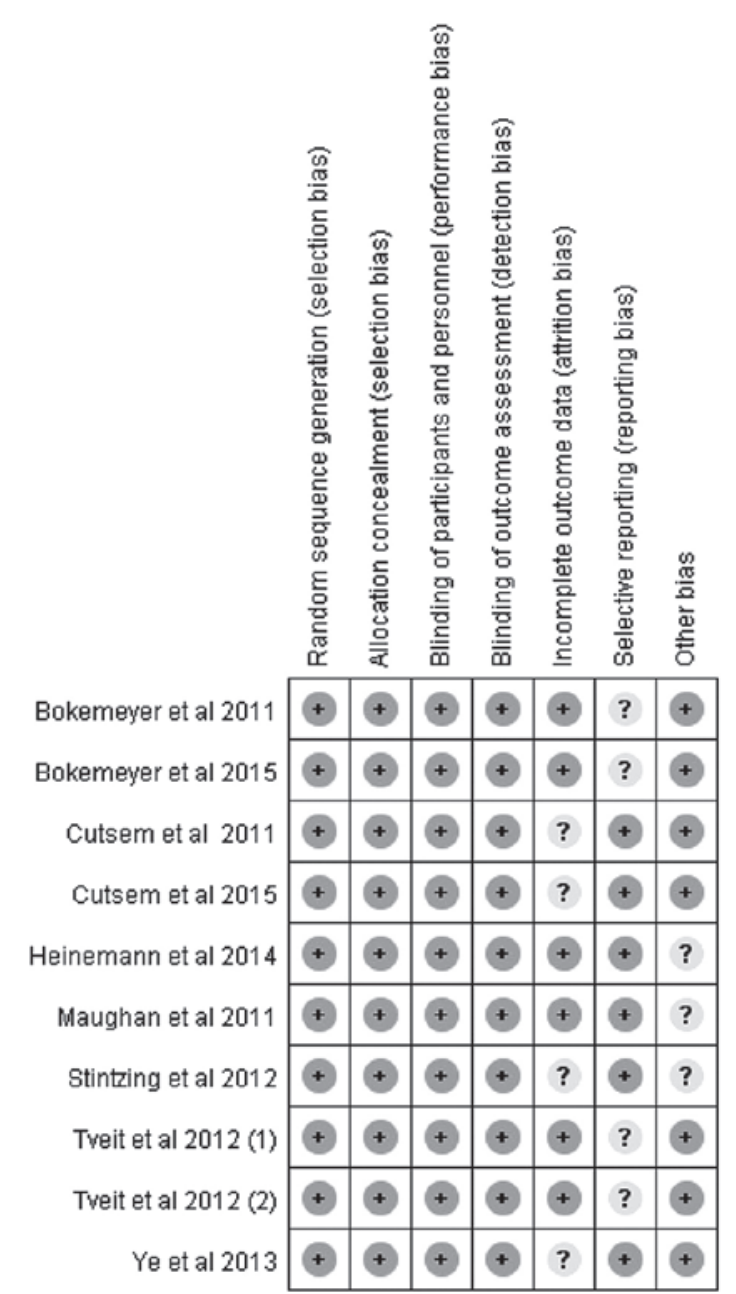

Figure 2. Risk of bias summary. 


\begin{tabular}{|c|c|c|c|c|c|c|c|c|c|}
\hline Study or Subqroup & log[Hazard Ratio] & SE & Weight & $\begin{array}{l}\text { Hazard Ratio } \\
\text { IV. Fixed. } 95 \% \mathrm{CI}\end{array}$ & & $\begin{array}{r}\text { Hazard } \\
\text { IV. Fixed. }\end{array}$ & $\begin{array}{l}\text { Ratio } \\
1.95 \% \mathrm{Cl}\end{array}$ & & \\
\hline Bokemeyer et al 2015 & -0.1508 & 0.1837 & $6.6 \%$ & $0.86[0.60,1.23]$ & & $\longrightarrow$ & E & & \\
\hline Cutsem et al 2015 & -0.2231 & 0.0905 & $27.2 \%$ & $0.80[0.67,0.96]$ & & $\rightarrow-$ & & & \\
\hline Heinemann et al 2014 & -0.2614 & 0.1105 & $18.2 \%$ & $0.77[0.62,0.96]$ & & $\rightarrow-$ & & & \\
\hline Maughan et al 2011 & -0.0387 & 0.0859 & $30.2 \%$ & $0.96[0.81,1.14]$ & & $\rightarrow$ & - & & \\
\hline Tveit et al 2012 (1) & 0.131 & 0.1807 & $6.8 \%$ & $1.14[0.80,1.62]$ & & 7 & & & \\
\hline Tveit et al 2012 (2) & 0.077 & 0.1726 & $7.5 \%$ & $1.08[0.77,1.51]$ & & & & & \\
\hline Ye et al 2013 & -0.6162 & 0.2513 & $3.5 \%$ & $0.54[0.33,0.88]$ & & 一 & & & \\
\hline Total $(95 \% \mathrm{Cl})$ & & & $100.0 \%$ & $0.87[0.79,0.96]$ & & $\nabla$ & & & \\
\hline \multicolumn{5}{|c|}{$\begin{array}{l}\text { Heterogeneity: } C h i^{2}=10.86, d f=6(P=0.09) ; I^{2}=45 \% \\
\text { Test for overall effect: } Z=2.90(P=0.004)\end{array}$} & $0.1 \quad 0.2$ & 0.5 & 2 & 5 & 10 \\
\hline
\end{tabular}

Figure 3. Comparison of cetuximab-based treatment group with the control group for KRAS-wild-type patients in terms of the overall survival. CI, confidence interval; SE, standard error of the mean.

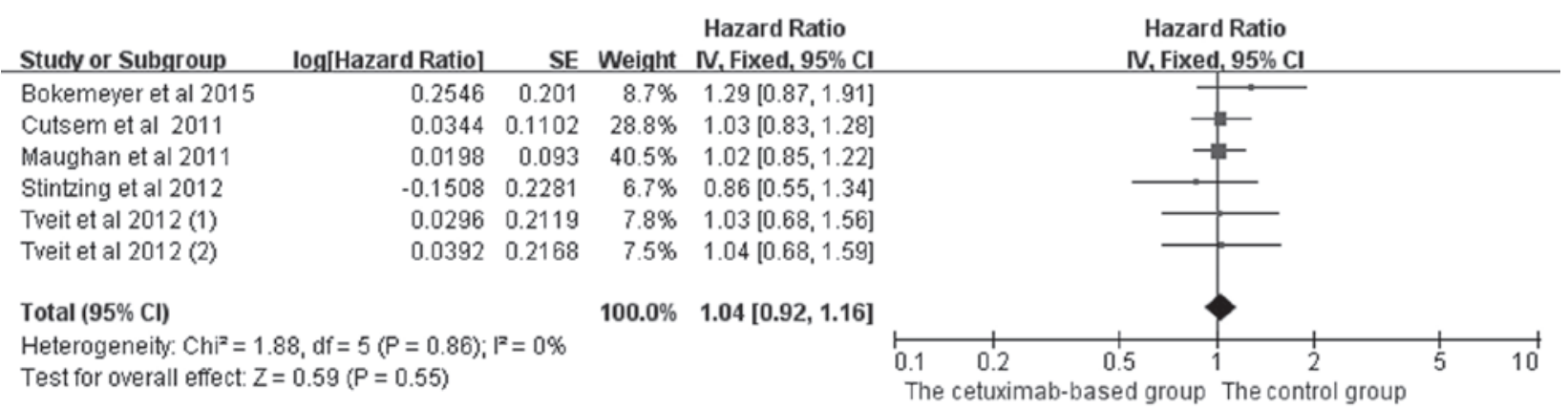

Figure 4. Comparison of cetuximab-based treatment group with the control group for KRAS-mutant patients in terms of the overall survival. CI, confidence interval; SE, standard error of the mean.

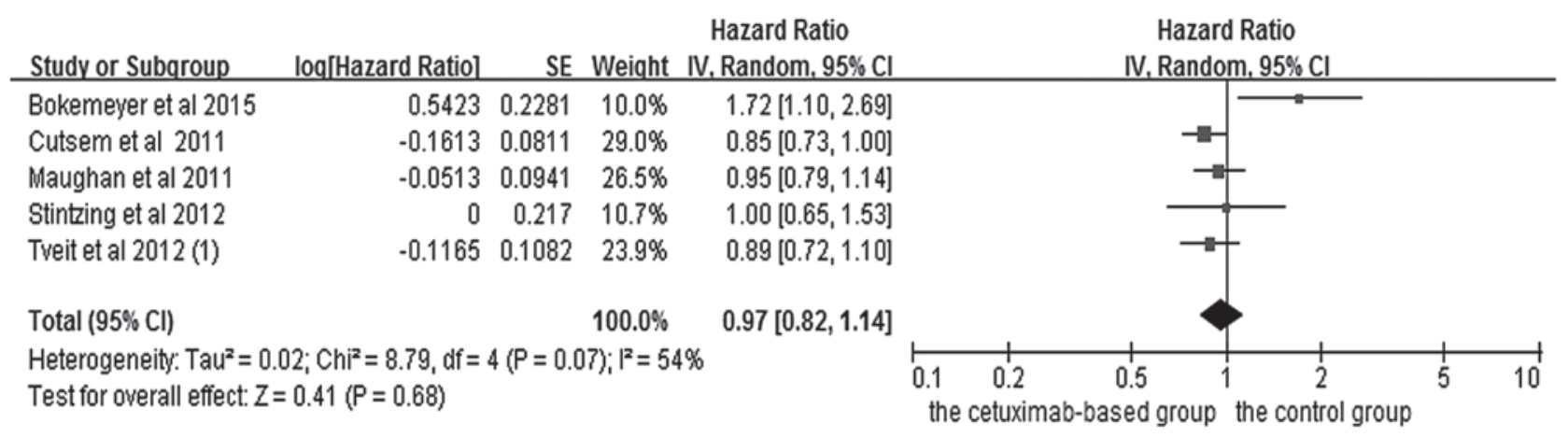

Figure 5. Comparison of cetuximab-based treatment group with the control group for KRAS-mt patients in terms of the progression free survival. CI, confidence interval; SE, standard error of the mean.

were updated analyses of the CRYSTAL trial (15), wherein the authors reported on an updated analysis of the larger cohort, as well as novel data on the impact of tumor BRAF and RAS mutations, respectively, among KRAS-wild type tumors on the clinical outcome $(2,16)$. The AIO KRK-0306 trial (17) and FIRE-3 (18) compared the treatment efficacy of irinotecan/5-fluorouracil/leucovorin (FOLFIRI) combined with bevacizumab or cetuximab, which is a first-line treatment for mCRC. The NORDIC-VII (5) multicenter phase III trial investigated the efficacy of cetuximab when added to bolus fluorouracil/folinic acid and oxaliplatin ('Nordic FLOX'), administered continuously or intermittently. Patients were randomly assigned to receive either standard Nordic
FLOX (arm A), cetuximab and FLOX (arm B), or cetuximab combined with intermittent FLOX (arm C). The predominant comparison was made between arms A and B. Arms B vs. A, and arms C vs. arms A, were defined as Tveit 1 and Tveit 2, respectively. Of the seven articles, the study by Ye et al (19) was the only one that assessed the effects of cetuximab plus chemotherapy as a first-line treatment, which was restricted to unresectable colorectal liver metastases. The remaining two studies by Bokemeyer et al $(3,20)$ were randomized phase II studies, which reported an updated analysis based on the OPUS study: These updated retrospective analyses comparatively investigated efficacy in the patient subgroups, defined according to the BRAF and RAS mutation status. 


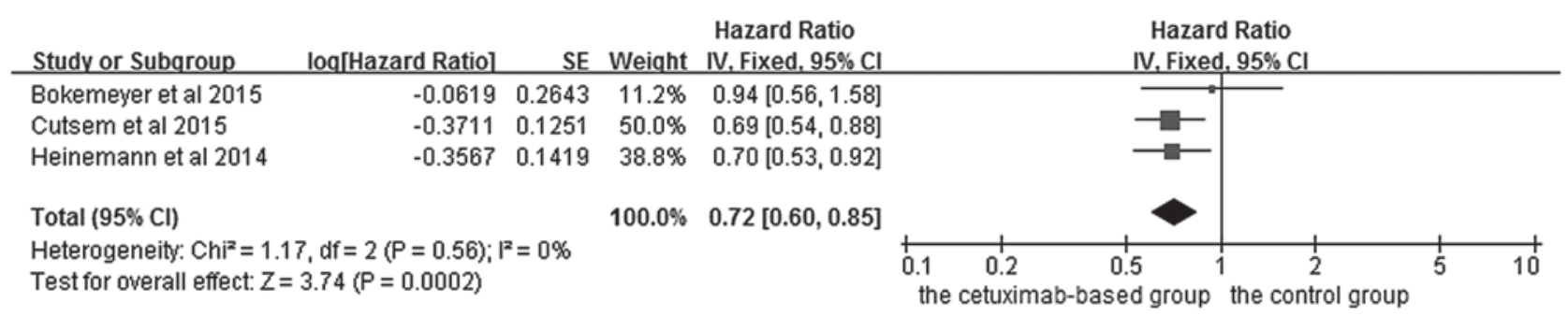

Figure 6. Forest plot of overall survival between cetuximab-based therapy and control in KRAS-wild-type/RAS-wild-type carriers. CI, confidence interval; $\mathrm{SE}$, standard error of the mean.

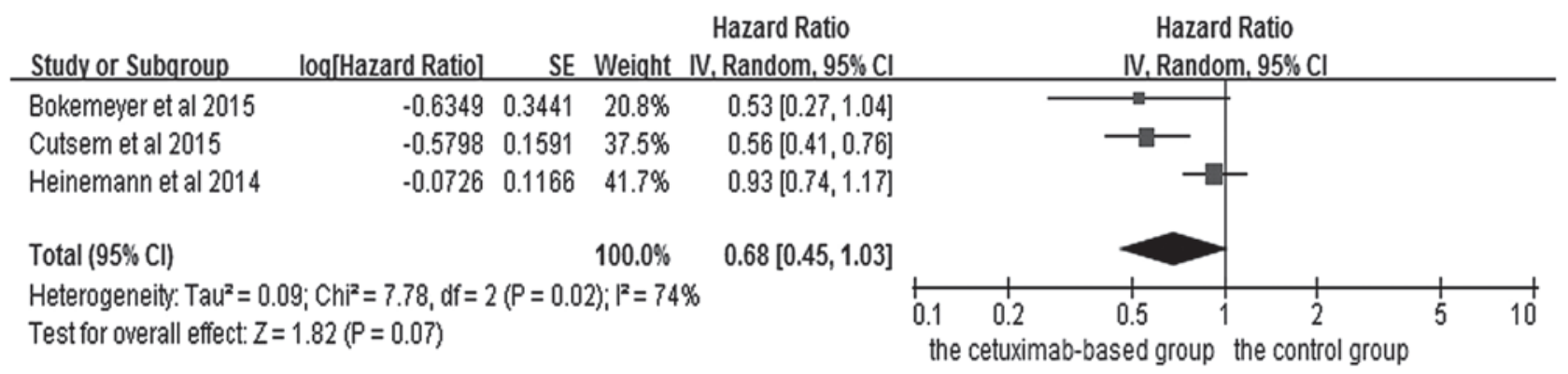

Figure 7. Forest plot of the progression free survival between cetuximab-based therapy and control in KRAS-wild-type/RAS-wild-type carriers. CI, confidence interval; SE, standard error of the mean.

A pooled analysis of OS and PFS between cetuximab-based therapy and chemotherapy \pm other targeted agents in KRAS exon 2 wild-type patients was performed.

The effects of cetuximab-based treatment on OS are shown in Fig. 3. OS data were available in six RCTs $(4,5,16,18-20)$. The aggregated results suggested that there was a significant OS benefit from cetuximab-based chemotherapy $(\mathrm{HR}=0.87$, 95\% $\mathrm{CI}=0.79-0.96, \mathrm{Z}=2.91, \mathrm{P}=0.004)$. All six $\mathrm{RCTs}$ reported data concerning PFS. However, even though the pooled analysis of PFS with an $\mathrm{I}^{2}$ value of $77 \%$ was considered to have high heterogeneity, the randomized-effects model was not available to be used.

Pooled analysis of OS and PFS between cetuximab-based therapy and chemotherapy \pm other targeted agents in KRAS exon 2 mutation-type patients. In the analysis of OS and PFS in patients with $\mathrm{mCRC}$ treated with chemotherapy, five studies $(2,4,5,17,20)$ were included, and the data are shown in Figs. 4 and 5. Since PFS was not an appropriate end point for the stop-and-go principle in the NORDIC-VII trial (5), comparisons including arm $\mathrm{C}$ were of interest primarily for OS. The OS $(\mathrm{HR}=1.04,95 \% \mathrm{CI}=0.92-1.16, \mathrm{Z}=0.59, \mathrm{P}=0.55)$ and PFS $(\mathrm{HR}=0.97,95 \% \mathrm{CI}=0.82-1.14, \mathrm{Z}=0.41, \mathrm{P}=0.68)$ benefits were not identified in the combined treatments.

Subgroup analysis of efficacy according to RAS mutation status in KRAS exon 2 wild-type patients. The effects of cetuximab-based chemotherapy treatment on OS and PFS in KRAS exon 2 wild-type and other RAS-mutant subgroup patients are shown in Figs. 6-9. OS and PFS data were available for three trials $(16,18,20)$. These individuals with wild-type KRAS exon 2 were divided into two subgroups: The 'all RAS wild-type' subgroup (no mutations in exons 2, 3 and 4 for either KRAS or NRAS), and the 'new RAS mutant' subgroup (wild-type for KRAS exon 2, but with a KRAS mutation in exons 3 or 4 and/or a NRAS mutation in exons 2,3 or 4). A significant OS benefit of cetuximab-based chemotherapy was evident in patients without any RAS mutations $(\mathrm{HR}=0.72,95 \% \mathrm{CI}=0.60-0.85, \mathrm{Z}=3.74$, $\mathrm{P}=0.0002)$, although not for $\mathrm{PFS}(\mathrm{HR}=0.68,95 \% \mathrm{CI}=0.45-1.03$, $\mathrm{Z}=1.82, \mathrm{P}=0.07)$. For the KRAS exon 2 wild-type with other RAS mutations, KRAS-wild/RAS-mutant tumors, no significant differences in the cetuximab-based treatment effects were observed in either PFS ( $\mathrm{HR}=1.19,95 \% \mathrm{CI}=0.56-2.54, \mathrm{Z}=0.45$, $\mathrm{P}=0.65)$ or $\mathrm{OS}(\mathrm{HR}=1.19,95 \% \mathrm{CI}=0.81-1.74, \mathrm{Z}=0.88, \mathrm{P}=0.38)$. In other words, adding cetuximab to the treatment for patients with KRAS exon 2 wild-type/other RAS-mutants did not improve PFS and OS compared with the control groups.

Subgroup analysis of efficacy according to BRAF mutation status in KRAS exon 2 wild-type patients. Three RCTs reported data of KRAS and BRAF tumor mutation status. However, not all the studies reported available data on OS and PFS, so it was therefore not possible to perform meta-analysis. The CRYSTAL trial (2) reported PFS (median, 8.0 vs. 5.6 months, $\mathrm{HR}=0.934, \mathrm{P}=0.87$ ) and $\mathrm{OS}$ (median, 14.1 vs. 10.3 months, $\mathrm{HR}=0.908, \mathrm{P}=0.74$ ) in patients with KRAS exon 2 wild-type/BRAF mutations who were treated with FOLFIRI plus cetuximab, although no statistical significance was identified. In addition, no trial was concerned with independent treatment for patients with BRAF mutations. Thus, with the current data, the BRAF mutation status cannot be a predictive factor for treatment outcomes of cetuximab plus FOLFIRI. KRAS exon 2 wild-type/BRAF wild-type patients treated with FOLFOX-4 plus cetuximab demonstrated marked improvements in PFS.

In the OPUS trial (3), only a small number of patients with mutations in the RAS gene were identified. For patients with the KRAS exon 2 wild-type/BRAF mutant ( $n=11)$, OS was prolonged in those receiving cetuximab plus FOLFOX-4 compared with those receiving FOLFOX-4 therapy alone 


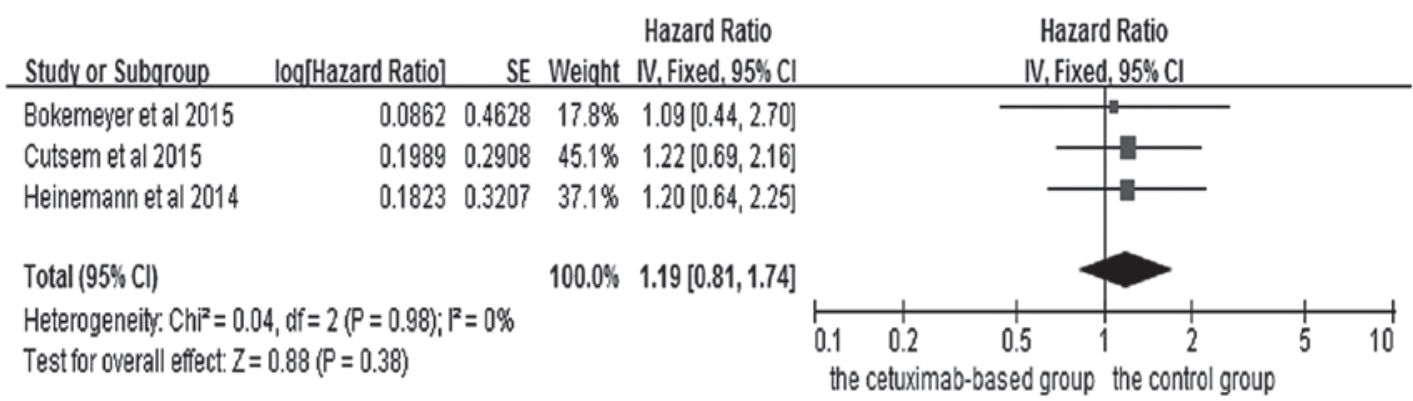

Figure 8. Forest plot of overall survival between cetuximab-based therapy and control in KRAS-wild-type/RAS-mutant carriers. CI, confidence interval; SE, standard error of the mean.

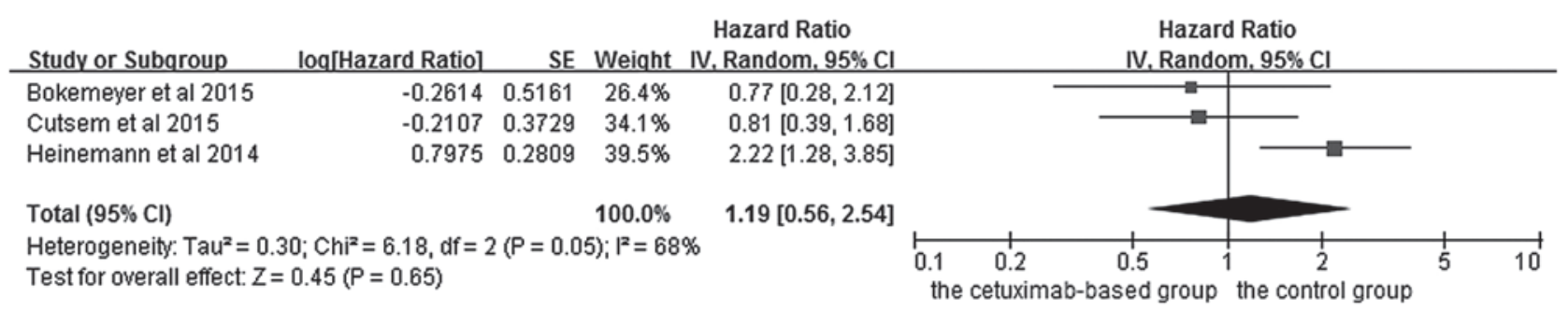

Figure 9. Forest plot of progression free survival between cetuximab-based therapy and control in KRAS-wild-type/RAS-mutant carriers. CI, confidence interval; SE, standard error of the mean.

(median, 20.7 vs. 4.4 months). This difference should be interpreted cautiously since, given the small sample size, no definitive conclusions concerning possible predictive or prognostic utility may be reached. In the COIN trial (4), the median OS was shorter in patients with BRAF mutations ( $n=102,8.8$ months) compared with those with both the BRAF wild-type and KRAS mutations ( $\mathrm{n}=548,14.4$ months) or NRAS mutations ( $\mathrm{n}=38,13.8$ months). The median PFS ranged from 5.6 months in patients with BRAF mutations, to 9.0 months for those with the wild type of all of KRAS, NRAS and BRAF.

\section{Discussion}

The predominant purpose of the meta-analysis performed in the present study was to critically evaluate the efficacy of adding cetuximab to chemotherapy in patient subgroups defined according to the RAS and BRAF gene type. In the meta-analysis, an OS benefit was observed in patients with KRAS exon 2 wild-type tumors ( $\mathrm{HR}=0.87,95 \% \mathrm{CI}=0.79-0.96$, $\mathrm{Z}=2.90, \mathrm{P}=0.004)$ and wild-type $\mathrm{KRAS} / \mathrm{RAS}$ patients $(\mathrm{HR}=0.72,95 \% \mathrm{CI}=0.60-0.85, \mathrm{Z}=3.74, \mathrm{P}=0.0002)$. In contrast, in patients carrying KRAS mutations, no matter whether in exon 2, exon 3 or 4 and/or a NRAS mutation in exons 2, 3 or 4, no evidence was identified of a benefit associated with cetuximab according to PFS and OS. In RAS subgroups $(16,18,20)$, patients with the KRAS/RAS-wild-type had a longer median OS time compared with the KRAS exon 2 wild-type. There are also additional trials [CALGB/SWOG 80405 (21); NORDIC VII (22)] that could potentially be retrospectively analyzed to determine whether there was a beneficial effect when cetuximab was combined with the KRAS exon 2 wild-type patients.

A potential direction of future study would be to evaluate individual RAS mutations in order to understand whether cetuximab efficacy varies among mutations. Several clinical studies have demonstrated that $\mathrm{mCRC}$ patients with the BRAF V600E mutation appear to have a poor prognosis. However, the association between the BRAF V600E mutation and the outcome of cetuximab-based treatment in the first-line treatment for patients with mCRC remains controversial $(2,3)$. To date, only two meta-analytical studies have been performed on the BRAF mutation status of HRs for PFS and OS, and this offers the explanation as to why meta-analysis of the BRAF mutation on the survival of patients with mCRC treated with cetuximab was not performed. For the CRYSTAL (2) and the COIN (4) trials, the advantage of the efficacy of the cetuximab-based chemotherapy is restricted to KRAS exon 2 wild-type/BRAF wild-type patients. In the OPUS trial (3), the results for the KRAS exon 2 wild-type/BRAF wild-type patients were very similar to those of the KRAS exon 2 wild-type patients receiving cetuximab plus FOLFOX-4, and were associated with significant improvements in the overall response rate and PFS. However, the sample size of patients with mutations in this gene was too small, analysis of the outcomes may have been influenced by imbalances in prognostic variables, and no definitive conclusions concerning possible predictive or prognostic utility may be reached. Analyses of larger numbers of patients are required to fully explore the biomarker potential of the BRAF mutation status for mCRC. Previously, patients with BRAF-mutant CRC have had an extremely poor prognosis compared with BRAF-wild-type patients, which has been subsequently confirmed by meta-analysis $(23,24)$. Bokemeyer et al (12) performed an analysis of the CRYSTAL and OPUS studies, and the objective of this pooled analysis was to investigate the efficacy of adding cetuximab to standard first-line chemotherapy according to KRAS and BRAF mutation status. This analysis collected data, including OS, PFS and the best overall response rate in 845 patients with KRAS wild-type receiving cetuximab plus chemotherapy. The cetuximab-based 
therapy significantly prolonged $\mathrm{OS}(\mathrm{HR}=0.81, \mathrm{P}=0.0062)$ and PFS (HR=0.66, $\mathrm{P}<0.001)$. The prognosis was worse in each treatment arm for patients with BRAF mutations compared with those with the BRAF wild-type. However, given the small sample size of BRAF mutation carriers, this result may not be entirely reliable. Taken together, BRAF mutations do not appear to be a predictive biomarker in current meta-analysis, but they do have predictive value for poor prognosis.

The meta-analysis performed in the present study had certain limitations. First, the trial results included in this meta-analysis were extracted from published data, rather than being based on data of the individual patients. Secondly, only studies with full published text were included in this analysis: All presentations at conferences were excluded. It is possible that the results of the full publication may differ from conference presentations due to updating of the data. Finally, inevitable variations existed among the studies, including the study design, basic therapies, follow-up intervals and type of therapy. All these factors could potentially affect the results of the meta-analysis.

In conclusion, the meta-analysis performed in the present study indicated that adding cetuximab to chemotherapy significantly improves OS in patients with $\mathrm{mCRC}$ who lack any RAS mutations (i.e., either in the KRAS exon 2 or any other RAS mutation). Individuals who carry the KRAS exon 2 wild-type, but who also have any novel type of RAS mutation, receive distinctly less benefit from cetuximab-based treatment compared with those without any RAS mutations. This meta-analysis also reveals that the BRAF V600E mutation may be associated with a poorer response and worse survival rates in wild-type KRAS mCRC patients treated with cetuximab. Given the limited number of studies included in the present meta-analysis, our findings need to be further explored and verified in larger randomized studies. Furthermore, all-RAS mutation and BRAF testing should be undertaken prior to the administration of cetuximab in order to obtain valuable prognostic and predictive information that may drive treatment decisions.

\section{References}

1. Jemal A, Bray F, Center MM, Ferlay J, Ward E and Forman D: Global cancer statistics. CA Cancer J Clin 61: 69-90, 2011.

2. Van Cutsem E, Köhne CH, Láng I, Folprecht G, Nowacki MP, Cascinu S, Shchepotin I, Maurel J, Cunningham D, Tejpar S, et al: Cetuximab plus irinotecan, fluorouracil and leucovorin as first-line treatment for metastatic colorectal cancer: Updated analysis of overall survival according to tumor KRAS and BRAF mutation status. J Clin Oncol 29: 2011-2019, 2011.

3. Bokemeyer C, Bondarenko I, Hartmann JT, de Braud F, Schuch G, Zubel A, Celik I, Schlichting M and Koralewski P: Efficacy according to biomarker status of cetuximab plus FOLFOX-4 as first-line treatment for metastatic colorectal cancer: The OPUS study. Ann Oncol 22: 1535-1546, 2011.

4. Maughan TS, Adams RA, Smith CG, Meade AM, Seymour MT, Wilson RH, Idziaszczyk S, Harris R, Fisher D, Kenny SL, et al: Addition of cetuximab to oxaliplatin-based first-line combination chemotherapy for treatment of advanced colorectal cancer: Results of the randomised phase 3 MRC COIN trial. Lancet 377: 2103-2114, 2011

5. Tveit KM, Guren T, Glimelius B, Pfeiffer P, Sorbye H, Pyrhonen S, Sigurdsson F, Kure E, Ikdahl T, Skovlund E, et al: Phase III trial of cetuximab with continuous or intermittent fluorouracil, leucovorin and oxaliplatin (Nordic FLOX) versus FLOX alone in first-line treatment of metastatic colorectal cancer: The NORDIC-VII study. J Clin Oncol 30: 1755-1762, 2012.
6. Bokemeyer C, Bondarenko I, Makhson A, Hartmann JT, Aparicio J, de Braud F, Donea S, Ludwig H, Schuch G, Stroh C, et al: Fluorouracil, leucovorin and oxaliplatin with and without cetuximab in the first-line treatment of metastatic colorectal cancer. J Clin Oncol 27: 663-671, 2009.

7. Douillard JY, Siena S, Cassidy J, Tabernero J, Burkes R, Barugel M, Humblet Y, Bodoky G, Cunningham D, Jassem J, et al: Final results from PRIME: Randomized phase III study of panitumumab with FOLFOX4 for first-line treatment of metastatic colorectal cancer. Ann Oncol 25: 1346-1355, 2014.

8. Price TJ, Peeters M, Kim TW, Li J, Cascinu S, Ruff P, Suresh AS, Thomas A, Tjulandin S, Zhang K, et al: Panitumumab versus cetuximab in patients with chemotherapy-refractory wild-type KRAS exon 2 metastatic colorectal cancer (ASPECCT): A randomised, multicentre, open-label, non-inferiority phase 3 study. Lancet Oncol 15: 569-579, 2014.

9. Cancer Genome Atlas Network: Comprehensive molecular characterization of human colon and rectal cancer. Nature 487: 330-337, 2012.

10. De Roock W, Claes B, Bernasconi D, De Schutter J, Biesmans B, Fountzilas G, Kalogeras KT, Kotoula V, Papamichael D, Laurent-Puig P, et al: Effects of KRAS, BRAF, NRAS and PIK3CA mutations on the efficacy of cetuximab plus chemotherapy in chemotherapy-refractory metastatic colorectal cancer: A retrospective consortium analysis. Lancet Oncol 11: 753-762, 2010.

11. Douillard JY, Oliner KS, Siena S, Tabernero J, Burkes R, Barugel M, Humblet Y, Bodoky G, Cunningham D, Jassem J, et al: Panitumumab-FOLFOX4 treatment and RAS mutations in colorectal cancer. N Engl J Med 369: 1023-1034, 2013.

12. Bokemeyer C, Van Cutsem E, Rougier P, Ciardiello F, Heeger S, Schlichting M, Celik I and Köhne CH: Addition of cetuximab to chemotherapy as first-line treatment for KRAS wild-type metastatic colorectal cancer: Pooled analysis of the CRYSTAL and OPUS randomised clinical trials. Eur J Cancer 48: 1466-1475, 2012.

13. Higgins JP and Thompson SG: Quantifying heterogeneity in a meta-analysis. Stat Med 21: 1539-1558, 2002.

14. Higgins JP, Thompson SG, Deeks JJ and Altman DG: Measuring inconsistency in meta-analyses. BMJ 327: 557-560, 2003.

15. Van Cutsem E, Köhne CH, Hitre E, Zaluski J, Chang Chien CR, Makhson A, D'Haens G, Pintér T, Lim R, Bodoky G, et al: Cetuximab and chemotherapy as initial treatment for metastatic colorectal cancer. N Engl J Med 360: 1408-1417, 2009.

16. Van Cutsem E, Lenz HJ, Köhne CH, Heinemann V, Tejpar S, Melezínek I, Beier F, Stroh C, Rougier P, van Krieken JH and Ciardiello F: Fluorouracil, leucovorin and irinotecan plus cetuximab treatment and RAS mutations in colorectal cancer. J Clin Oncol 33: 692-700, 2015.

17. Stintzing S, Fischer von Weikersthal L, Decker T, Vehling-Kaiser U, Jäger E, Heintges T, Stoll C, Giessen C, Modest DP, Neumann J, et al: FOLFIRI plus cetuximab versus FOLFIRI plus bevacizumab as first-line treatment for patients with metastatic colorectal cancer-subgroup analysis of patients with KRAS: Mutated tumours in the randomised German AIO study KRK-0306. Ann Oncol 23: 1693-1699, 2012.

18. Heinemann V, von Weikersthal LF, Decker T, Kiani A, Vehling-Kaiser U, Al-Batran SE, Heintges T, Lerchenmüller C, Kahl C, Seipelt G, et al: FOLFIRI plus cetuximab versus FOLFIRI plus bevacizumab as first-line treatment for patients with metastatic colorectal cancer (FIRE-3): A randomised, open-label, phase 3 trial. Lancet Oncol 15: 1065-1075, 2014

19. Ye LC, Liu TS, Ren L, Wei Y, Zhu DX, Zai SY, Ye QH, Yu Y, $\mathrm{Xu} \mathrm{B}$, Qin XY and Xu J: Randomized controlled trial of cetuximab plus chemotherapy for patients with KRAS wild-type unresectable colorectal liver-limited metastases. J Clin Oncol 31: 1931-1938, 2013.

20. Bokemeyer C, Köhne CH, Ciardiello F, Lenz HJ, Heinemann V, Klinkhardt U, Beier F, Duecker K, van Krieken JH and Tejpar S: FOLFOX4 plus cetuximab treatment and RAS mutations in colorectal cancer. Eur J Cancer 51: 1243-1252, 2015.

21. Venook AP, Niedzwiecki D, Lenz HJ, Innocenti F, Mahoney MR, O'Neil BH, Shaw JE, Polite BN, Hochster HS, Atkins AN, et al: CALGB/SWOG 80405: Phase III trial of irinotecan/5-FU/leucovorin (FOLFIRI) or oxaliplatin/5-FU/leucovorin (mFOLFOX6) with bevacizumab (BV) or cetuximab (CET) for patients (pts) with KRAS wild-type (wt) untreated metastatic adenocarcinoma of the colon or rectum (MCRC). ASCO Meeting Abstracts 32: LBA3, 2014. 
22. Tveit K, Guren T, Glimelius B, Pfeiffer P, Sorbye H, Pyrhonen S, Kure E, Ikdahl T, Skovlund T and Christoffersen T: Randomized phase III study of 5-fluorouracil/folinate/oxaliplatin given continuously or intermittently with or without cetuximab, as first-line treatment of metastatic colorectal cancer: The NORDIC VII study (NCT00145314), by the Nordic colorectal cancer biomodulation group. ASCO Meeting Abstracts 29: 365, 2011.

23. Pietrantonio F, Petrelli F, Coinu A, Di Bartolomeo M, Borgonovo K, Maggi C, Cabiddu M, Iacovelli R, Bossi I, Lonati $\mathrm{V}$, et al: Predictive role of BRAF mutations in patients with advanced colorectal cancer receiving cetuximab and panitumumab: A meta-analysis. Eur J Cancer 51: 587-594, 2015.
24. Cui D, Cao D, Yang Y, Qiu M, Huang Y and Yi C: Effect of BRAF V600E mutation on tumor response of anti-EGFR monoclonal antibodies for first-line metastatic colorectal cancer treatment: A meta-analysis of randomized studies. Mol Biol Rep 41: 1291-1298, 2014. 